\title{
Unconscious pop-out: attentional capture by unseen feature singletons only when top-down attention is available
}

\author{
Po-Jang Hsieh ${ }^{\star}, 1,2$, Jaron T. Colas ${ }^{2}$, and Nancy Kanwisher ${ }^{2}$ \\ ${ }^{1}$ Duke-NUS Graduate Medical School, Singapore 169857 \\ ${ }^{2}$ Department of Brain and Cognitive Sciences, McGovern Institute, MIT, Cambridge, MA 02139, \\ USA
}

\begin{abstract}
Visual "pop-out" occurs when a unique visual target (e.g. a feature singleton) is present among a set of homogeneous distractors. However, the role of visual awareness in this process remains unclear. Here we show that, even though subjects were not aware of a suppressed pop-out display, their subsequent performance on an orientation discrimination task was significantly better at the pop-out location than at a control location. These results indicate that visual awareness of a feature singleton is not necessary for it to attract attention. Furthermore, our results show that the subliminal pop-out effect disappeared when subjects diverted their attention toward an RSVP task while viewing the same subliminal pop-out display, suggesting that the availability of top-down attention is necessary for the subliminal pop-out effect, and that the cognitive processes underlying attention and awareness are somewhat independent.
\end{abstract}

\begin{abstract}
Visual "pop-out" refers to the phenomenon in which a unique visual target (e.g. a feature singleton) can be rapidly detected among a set of homogeneous distractors (Treisman, 1985; Wolfe, 1994). Here we inquire about the roles of visual awareness and attention in this process. Specifically, what actually makes a feature singleton pop out? Is awareness of the feature singleton necessary for the stimulus to attract attention? Or can a feature singleton be processed and summon attention without the subject ever being aware of the stimulus? ${ }^{1}$

We investigated (1) whether visual awareness of a feature singleton is necessary for that stimulus to capture attention and (2) whether top-down attention is necessary to elicit such a subliminal response. Using the continuous flash suppression paradigm (Tsuchiya and Koch, 2005), we presented a pop-out display (a feature singleton among several distractors) subliminally and demonstrated that, even though subjects were not aware of the display, their subsequent performance on an orientation discrimination task was significantly better at the pop-out location than at a control location (Experiment 1). Furthermore, we showed that this effect disappeared when subjects diverted their attention toward a rapid serial visual presentation (RSVP) task while viewing the same subliminal pop-out display (Experiment 2). Together, these findings suggest that (1) awareness of a feature singleton is not necessary for it to summon attention and that (2) top-down attention is necessary for this subliminal effect to occur.
\end{abstract}

\footnotetext{
*To whom correspondence should be addressed. hsieh.pj@gmail.com.

${ }^{1}$ Awareness is operationalized here as reportability (Dehaene et al., 2006). Top-down attention has been related to spatial, featural, temporal, and object-based variants of attention behaviorally, and neuronal 'versions' of top-down attention also include 'shrinking receptive fields' (Bundesen et al., 2005). For our purposes, top-down attention here only refers to the spatial and featural variants of attention.
} 


\section{Method}

\section{Participants}

Twelve, sixteen, nine, eleven, and five healthy adult volunteers with normal depth perception and normal or corrected-to-normal visual acuity were run in Experiments 1a, 1b, $1 \mathrm{c}, 2 \mathrm{a}$, and $2 \mathrm{~b}$, respectively. All subjects for all experiments gave informed consent within a protocol approved by the Massachusetts Institute of Technology and were paid 5 dollars for a session lasting approximately 30 minutes (Experiments 1a, 1b, 1c, and 2a) or 40 dollars for a session lasting approximately 4 hours (Experiment $2 b$ ).

\section{Stimuli and Procedures}

The stimulus configuration and experimental procedures used in Experiment 1a are shown in Figure 1. Participants viewed dichoptic images through a mirror stereoscope in a dark room. The fixation spot was white and subtended $0.27 \times 0.27$ degrees of visual angle on a black background. The visual stimuli for one eye during the beginning of each trial included twenty colored Gabor patches. Each of these subtended $1.1 \times 1.1 \mathrm{deg}$ and had a spatial frequency of $5.5 \mathrm{cpd}$ and a horizontal orientation. Twelve Gabors were arranged in a circle around the fixation point with a radius of $4.2 \mathrm{deg}$. Four Gabors were located at the corners of a square that was centered at the fixation point and had sides of length $8.9 \mathrm{deg}$. The remaining four were located $2.1 \mathrm{deg}$ above, below, and to the left and right of the fixation point. The default color for all of these Gabors was green. In each trial one of the twelve radial Gabors was randomly selected to be red (as a feature singleton). The luminance levels of red and green were subjectively matched before the experiments (Anstis and Cavanagh, 1983). Four identical dynamic Mondrian patterns were presented in the other eye with a refresh rate of $10 \mathrm{~Hz}$ to mask the four quadrants of a frame with sides of length $10.6 \mathrm{deg}$. Ten distinct Mondrians consisting of randomly generated red, blue, yellow, and white rectangles were generated before the experiment.

The initial phase of each trial lasted $2000 \mathrm{~ms}$, during which the contrast of all twenty Gabors increased linearly from $0 \%$ to $25 \%$. Following a blank inter-stimulus interval of $250 \mathrm{~ms}$, a nearly vertical white Gabor with $100 \%$ contrast was presented for $25 \mathrm{~ms}$ at either the location of the unique red Gabor or the diametrically opposed control location. The test Gabor was rotated 1.5 deg clockwise or counterclockwise, and the observer was required to press one of two buttons (two-alternative forced choice; $2 \mathrm{AFC}$ ) to indicate which orientation he or she perceived. At the end of every trial, subjects also indirectly indicated whether or not the Mondrians effectively masked the colored Gabors by reporting whether any of the quadrants appeared different. In the event that suppression was interrupted in 5 or more trials for a particular subject, his or her data would be excluded from the analysis because for these subjects who saw the "subliminal" stimulus too often, there might not be enough trials left and we can be less confident that the remaining trials are pure, so to be conservative, we excluded these subjects. (This occurred for 5 of 12 subjects in Experiment 1a, 4 of 16 subjects in Experiment 1b, 2 of 9 subjects in Experiment 1c, and 4 of 11 subjects in Experiment 2a.) For the remaining subjects, fewer than 5 trials with broken suppression were removed when calculating each subject's performance.

The eye in which the suppressed Gabors were presented alternated with every trial. The possible locations for assignment to a red Gabor (one of the twelve Gabors that were arranged in a circle around the fixation point), the orientation of the test Gabor (left or right tilt), and the location in which the test Gabor appeared (target or control) were all randomized and counterbalanced across the 48 trials in each block. Each participant completed one block. 
Subjects placed their chins on a chin rest for visual stabilization. The visual stimulator was a Dell workstation running Windows XP. The stimuli were presented with Vision Egg on a 20 -inch SONY CRT gamma-corrected monitor with a resolution of $1024 \times 768$ pixels and a frame rate of $100 \mathrm{~Hz}$.

The stimulus configuration and experimental procedures for Experiments $1 \mathrm{~b}$ and $1 \mathrm{c}$ were identical to those of Experiment 1a, except as follows. To investigate whether the presence of red in the Mondrians might affect the pop-out effect for a red singleton, the Mondrians used in Experiment $1 \mathrm{~b}$ were instead produced with only blue, yellow, and white rectangles. As in Experiment 1a, subjects were required to press one of two buttons (2AFC) to indicate which orientation he or she perceived. Additionally, subjects were required to indicate whether the masking was effective by reporting whether or not they perceived any colors other than those appearing in the Mondrians.

To provide a more stringent test of awareness of the pop-out display, we doubled the number of trials (to 96) in Experiment 1c and removed the pop-out display for half of them, such that only one eye received Mondrians and the other eye received no visual stimulus. At the end of each trial, the subject was required to first press one of two buttons (2AFC) to indicate which orientation he or she perceived, and then indicate whether the masking was effective by reporting whether or not they perceived any colors other than those appearing in the Mondrians. Additionally, subjects were required to guess (2AFC) if a pop-out display was presented during the initial phase, even if they reported that they did not perceive any colors other than those appearing in the Mondrians in response to the second question.

The stimulus configuration, experimental procedures, and data analysis for Experiment 2a were identical to those of Experiment 1a, except as follows. The initial phase of each trial lasted $9 \mathrm{~s}$, but the contrast of the suppressed Gabors still reached its peak of $25 \%$ by $2 \mathrm{~s}$ and remained at $25 \%$ thereafter. During this initial phase, a rapid serial visual presentation (RSVP) of the ten numerical digits was presented monocularly in front of the Mondrians at fixation. This random sequence was looped six times with a refresh rate of $6.67 \mathrm{~Hz}$. The digits were black and subtended up to $1.5 \times 2.3$ degrees of visual angle. The observer was required to press a button every time a probe (the digit " 5 ") appeared, which occurred three times per trial.

In Experiment 2b different inter-stimulus intervals (ISIs; 100, 150, 200, 250, and $300 \mathrm{~ms}$ ) between the offset of suppression and the onset of the test Gabor were further investigated. The stimulus configuration and experimental procedures utilized for the "attention available" blocks were otherwise similar to those of Experiment 1c. The duration of the test Gabor was increased to $50 \mathrm{~ms}$. In the beginning of each block, the maximum contrast of the suppressed Gabors was still assigned to $25 \%$. However, after each trial the contrast was instead adjusted dynamically according to whether or not suppression was broken, decreasing by $2 \%$ when suppression was broken or increasing by $1 \%$ otherwise. Such adjustment was made to make sure the contrast of the "subliminal" Gabor is around the breaking threshold so that it is strong enough to be effective. The stimulus configuration and experimental procedures utilized for the "no attention" blocks were likewise similar to those of Experiment 2a, but the initial suppression phase of each trial lasted only $2 \mathrm{~s}$ to parallel that of the "attention available" blocks. Additionally, the RSVP sequence was instead looped twice and with a refresh rate of $10 \mathrm{~Hz}$. To control for the generation of a motor response, subjects reported whether or not the RSVP probe appeared (2AFC) after the trial ended. Each subject completed two blocks of each condition (with or without attention), each of which included 480 trials. 


\section{Data analysis}

We computed the percentage of trials in which subjects responded correctly to the orientation of the test Gabor. In Experiments 1 and 2a a paired t-test was performed to compare these percentages for the two conditions (feature singleton location versus control location) across subjects. In Experiment $2 \mathrm{~b}$ a two-way repeated measures ANOVA (Gabor location $\times$ ISI) was performed within both the "attention available" blocks and the "no attention" blocks to test for significant main effects of location (i.e. singleton target versus control). A three-way omnibus ANOVA (block $\times$ location $\times$ ISI) was performed to test for a three-way interaction of block, location, and ISI. Finally, post-hoc paired t-tests between the effect sizes (target - control) of the two blocks were conducted for each ISI.

\section{Results}

\section{Experiment 1: Unseen feature singleton attracts attention}

Results in Figure 2a show the percentage of trials in which subjects responded correctly to the orientation of the test Gabor in Experiment 1a. Data averaged across subjects indicate that performance on the orientation discrimination task for the location of the feature singleton is significantly greater than that of the control location (mean effect size $=5.34 \%$, paired $\mathrm{t}(6)=4.51, \mathrm{p}=.004)$.

To control for a possible bias due to the presence of red in both the Mondrians and the feature singletons, the Mondrians used in Experiment $1 \mathrm{~b}$ were instead produced with only blue, yellow, and white rectangles. Results in Figure $2 \mathrm{~b}$ show the percentage of trials in which subjects responded correctly to the orientation of the test Gabor in Experiment $1 b$. Data averaged across subjects indicate that, as in Experiment 1a, performance on the orientation discrimination task for the location of the feature singleton is significantly greater than that of the control location (mean effect size $=6.31 \%$, paired $t(11)=2.25, \mathrm{p}=$. 046).

To provide a more stringent test of awareness of the pop-out display, we doubled the number of trials in Experiment 1c and removed the pop-out display for half of them. At the end of each trial, the subject was required to first press one of two buttons (2AFC) to indicate which orientation he or she perceived and then indicate whether the masking was effective by reporting whether or not they perceived any colors other than those appearing in the Mondrians, which were identical to those of Experiment 1b. Additionally, subjects were required to guess (2AFC) if a pop-out display was presented during the initial phase when they reported suppression in response to the second question. Results in Figure 3a show the percentage of trials in which subjects correctly guessed whether the pop-out display was present. Data averaged across subjects indicate that performance on this task is not significantly different from chance (paired $t(6)=0.827, p=.440$ ). Results in Figure $3 \mathrm{~b}$ show the percentage of trials in which subjects responded correctly to the orientation of the test Gabor. Data averaged across subjects indicate that, as in Experiments 1a and 1b, performance on the orientation discrimination task for the location of the feature singleton is significantly greater than that of the control location (mean effect size $=10.20 \%$, paired $\mathrm{t}(6)$ $=3.15, \mathrm{p}=.020)$.

\section{Experiment 2: Top-down attention is necessary for the subliminal pop-out effect}

In Experiment 2 we tested whether top-down attention is necessary for such a subliminal pop-out effect. The stimulus configuration, experimental procedures, and data analysis utilized for Experiment 2a were almost identical to those of Experiment 1a, except as follows. The initial phase of each trial lasted $9 \mathrm{~s}$, during which a rapid serial visual 
presentation (RSVP) of the ten numerical digits was presented at fixation. The observer was required to press a button every time a probe (the digit " 5 ") appeared.

Results in Figure 4a show the percentage of trials in which subjects responded correctly to the orientation of the test Gabor in Experiment 2a. Data averaged across subjects indicate that, unlike Experiments 1a, 1b, and 1c, performance on the orientation discrimination task for the location of the feature singleton is not significantly different from that of the control location (paired $\mathrm{t}(6)=0.770, \mathrm{p}=.471$ ). Moreover, the magnitude of the effect size between the two conditions is significantly greater for Experiment 1 than for Experiment 2a (mean effect size $=9.74 \%$, two-sample $\mathrm{t}(31)=2.76, \mathrm{p}=.009$ ).

One might argue that the subliminal pop-out effect disappeared in Experiment 2a not because top-down attention was unavailable when engaged with the RSVP task, but simply because the presentation duration was too long $(9 \mathrm{~s})$ for the pop-out effect to maintain. To test this possibility, we fixed the duration of the initial suppression phase ( 2 seconds) in Experiment $2 \mathrm{~b}$ for the "attention available" and "no attention" conditions. We additionally explored the influence of different ISIs (between the offset of suppression and the onset of the test Gabor) on the subliminal pop-out effect. Results in Figure 4b show the percentage of trials in which subjects responded correctly to the orientation of the test Gabor in Experiment $2 \mathrm{~b}$ as a function of the ISI for both the feature singleton location and the control location.

For the "attention available" blocks, analysis by means of a two-way repeated measures ANOVA (location $\times$ ISI) revealed a main effect of location-i.e., performance on the orientation discrimination task for the location of the feature singleton is significantly greater than that of the control location $(\mathrm{F}(1,4)=8.241, \mathrm{p}=0.045)$. (Neither the main effect of ISI $(\mathrm{F}(4,16)=2.867, \mathrm{p}=0.058)$, nor an interaction $(\mathrm{F}(4,16)=1.872, \mathrm{p}=0.165)$ reach significance.) For the "no attention" blocks, neither a significant main effect of location $(\mathrm{F}(1,4)=0.257, \mathrm{p}=0.639)$ or of ISI $(\mathrm{F}(4,16)=2.240, \mathrm{p}=0.110)$, nor a significant interaction were found $(\mathrm{F}(4,16)=2.765, \mathrm{p}=0.064)$.

Given that the above analyses suggest that an effect of location is present for the "attention available" block but not the "no attention" block (i.e. subliminal pop-out effect is only present in the "attentional available" blocks), we proceeded to further test this possibility by performing a three-way omnibus ANOVA (block $\times$ location $\times$ ISI) over all data. This ANOVA revealed a significant three-way interaction of block, location, and ISI $(F(4,16)=$ $3.985, p=0.020$ ), indicating an interaction of block and location over some ISIs more than others, and licensing additional post-hoc analyses at each ISI. We therefore performed posthoc paired t-tests over the effect sizes (target - control) between the two blocks at each ISI, and the results revealed a significant difference between the two blocks only for the $300 \mathrm{~ms}$ ISI $(t(4)=3.162, p=0.034)$ and not for the other ISIs $(t(4)<1.908, p>0.129)$. Thus, for the $300 \mathrm{~ms}$ ISI, we see the key interaction of block and location, indicating that visual awareness of a feature singleton is not necessary for it to attract attention, and that the availability of top-down attention is necessary for such subliminal pop-out effect.

\section{Discussion}

Here we demonstrated that a subliminal feature singleton enhances subjects' performance on an orientation discrimination task at the stimulus location, indicating that the feature singleton attracted attention even though observers were unaware of both the singleton itself and its distractors. This result is consistent with prior findings that stimuli that are not perceived consciously can nonetheless be perceptually analyzed (see Kouider and Dehaene (2007) for review) and can affect spatial attention (Astle et al., 2010; Jiang et al., 2006; Lin 
et al., 2009; McCormick, 1997). Our finding goes beyond these prior results insofar as we have established that a feature singleton can be processed and summon attention even when it is not perceived consciously. Many theories of visual search posit that there exists a preattentive processing stage that extracts featural contrasts from the visual scene (Itti \& Koch, 2001; Wolfe, 1994) and integrates them into a saliency/activation/priority map that guides spatial attention to the map's most intense location. Consistent with these models, the data of Experiment 1 demonstrate that attention can be shifted to the location of a feature singleton and induce increased orientation sensitivity, even if the feature singleton is presented subliminally.

The subliminal pop-out effect is in line with a previous finding (Zhaoping, 2008) which establishes that attention can be captured by supraliminal eye of origin singletons even when observers are unaware of the eye of origin of visual inputs. Our findings differ insofar as we demonstrated that, even when an observer was completely prevented from consciously perceiving a feature singleton (as opposed to perceiving a stimulus without awareness of its eye of origin), this suppressed feature singleton could nonetheless attract attention. In other words, our findings further suggest that the saliency map from one eye can be constructed even when the stimuli presented to that eye are completely suppressed from consciousness.

Moreover, our data from Experiment 2 also show that attentional recruitment by unseen feature singletons was not observed when subjects diverted their attention toward an RSVP task while viewing the same subliminal pop-out display. This finding suggests that top-down attention might be necessary for pre-attentive calculation of featural contrasts. This interpretation is consistent with prior findings showing that perceptual processing (e.g. cueing of spatial attention, the supraliminal pop-out effect, perceptual grouping, texturebased analysis, flicker perception, subliminal orientation processing) can be diminished when cognitive load is high (Ben-Av et al., 1992; Joseph et al., 1997; Lee et al., 2009; Moore and Egeth, 1997; Carmel et al., 2007; Santangelo et al., 2008; Baharami et al., 2008; but see Braun and Sagi, 1990, 1991; Egeth et al., 2008), and is in line with studies showing that top-down, feature-based attention can modulate the processing of invisible stimuli (Baharami et al., 2007; Kanai et al., 2006; Wyart and Tallon-Baudry, 2008). Together, these findings challenge the view that popout for visual attributes such as orientation, color, or size differences are processed 'preattentively' (Neisser, 1967; Treisman and Gelade, 1980) and instead suggest that top-down attention can be critical for the detection of some of these purportedly 'preattentive' features.

Alternatively, it might be the case that the calculation of featural contrast and the resulting saliency map are identical in both Experiments 1 and 2, but in Experiment 2 spatial attention is engaged so strongly at the center of the visual field that the weak saliency signal is not sufficient to capture attention. Consistent with this notion, Leber \& Egeth (2006) proposed that, in order for a salient singleton to automatically attract attention, observers have to engage a "singleton search mode". It is possible that observers were in such a search mode in Experiment 1, but not in Experiment 2, because they were completely engaged in the RSVP task in the latter case. Additionally, Belopolsky et al. (2007) introduced the concept of "size of the attentional window" (similar to a zoom lens model of spatial attention) and conclude that, in order for salient singletons to attract attention, the attentional window has to be distributed over the whole visual field, which is not the case in Experiment 2.

Although these possibilities need to be distinguished more finely in future studies, our findings nevertheless challenge the notion that attention and awareness are inextricably coupled (Mack and Rock; 1998; Merikle and Joordens, 1997; O'Regan and Noe, 2001).

To conclude, here we demonstrate a dissociation of attention and awareness in support of the view of the two processes as related but distinct phenomena that need not occur together and 
that entail distinct functions and neural mechanisms (Dehaene et al., 2006; Kentridge et al., 2004, 2008; Koch and Tsuchiya, 2006; Lamme, 2003; Naccache et al., 2002). This dissociation of attentional cueing and awareness also suggests that the neural substrates for bottom-up saliency reside at an early stage in the visual pathway before visual processing has reached sufficient awareness. This finding is consistent with the theory $(\mathrm{Li}, 2002)$ that pre-attentive computation in primary visual cortex creates a saliency map and inconsistent with others which imply that the saliency map results from summing various visual feature maps and therefore must reside in a higher brain region where neurons are no longer tuned to any low-level visual features (Koch and Ullman, 1985).

\section{References}

Anstis, S.; Cavanagh, P. Colour vision:Physiology and psychophysics. Mollon, JD.; Sharpe, LT., editors. New York: Academic Press; 1983. p. 156-166.

Astle DE, Nobre AC, Scerif G. Subliminally presented and stored objects capture attention. Journal of Neuroscience. 2010; 30:3567-3571. [PubMed: 20219990]

Bahrami B, Lavie N, Rees G. Attentional load modulates responses of human primary visual cortex to invisible stimuli. Current Biology. 2007; 17:509-513. [PubMed: 17346967]

Bahrami B, Carmel D, Walsh V, Rees G, Lavie N. Unconscious orientation processing depends on perceptual load. Journal of Vision. 2008; 8(3):1-10. [PubMed: 18484818]

Belopolsky AV, Zwaan L, Theeuwes J, Kramer AF. The size of an attentional window modulates attentional capture by color singletons. Psychon Bull Rev. 2007; 14(5):934-8. [PubMed: 18087962]

Ben-Av MB, Sagi D, Braun J. Visual attention and perceptual grouping. Perception \& Psychophysics. 1992; 52:277-294. [PubMed: 1408639]

Braun J, Sagi D. Vision outside the focus of attention. Perception \& Psychophysics. 1990; 48:45-58. [PubMed: 2377439]

Braun J, Sagi D. Texture-based tasks are little affected by a second task which requires peripheral or central attentive fixation. Perception. 1991; 20:483-500. [PubMed: 1771133]

Bundesen C, Habekost T, Kyllingsbaek S. A neural theory of visual attention: bridging cognition and neurophysiology. Psychol Rev. 2005; 112(2):291-328. [PubMed: 15783288]

Carmel DV, Rees G, Lavie N. Perceptual load modulates conscious flicker perception. Journal of Vision. 2007; 7(14):1-13. 14. [PubMed: 18217809]

Dehaene S, Naccache L, Le Clec HG, Koechlin E, Mueller M, Dehaene-Lambertz G, van de Moortele PF, Le Bihan D. Imaging unconscious semantic priming. Nature. 1998; 395:597-600. [PubMed: 9783584]

Dehaene $\mathrm{S}$, et al. Conscious, preconscious, and subliminal processing: a testable taxonomy. Trends Cogn Sci. 2006; 10:204-211. [PubMed: 16603406]

Egeth HE, Leonard CJ, Palomares M. The role of attention in subitizing: Is the magical number 1? Visual Cognition. 2008; 16:463-473.

Jiang Y, Costello P, Fang F, Huang M, He S. A gender- and sexual orientation-dependent spatial attention effect of invisible images. Proc Natl Acad Sci USA. 2006; 103(45):17048-17052. [PubMed: 17075055]

Joseph JS, Chun MM, Nakayama K. Attentional requirements in a "preattentive" feature search task. Nature. 1997; 387:805-807. [PubMed: 9194560]

Itti L, Koch C. Computational modelling of visual attention. Nature Reviews, Neuroscience. 2001; 2:194-203.

Kanai R, Tsuchiya N, Verstraten FA. The scope and limits of top-down attention in unconscious visual processing. Current Biology. 2006; 16:2332-2336. [PubMed: 17141615]

Kentridge RW, et al. Spatial attention speeds discrimination without awareness in blindsight. Neuropsychologia. 2004; 42:831-835. [PubMed: 15037061]

Kentridge RW, et al. Attended but unseen: Visual attention is not sufficient for visual awareness. Neuropsychologia. 2008; 46:846-869. 
Koch C, Ullman S. Shifts in selective visual attention: Towards the underlying neural circuitry. Human Neurobiology. 1985; 4:219-227. [PubMed: 3836989]

Koch C, Tsuchiya N. Attention and consciousness: two distinct brain processes. Trends in Cognitive Sciences. 2006; 11(1):16-22. [PubMed: 17129748]

Kouider S, Dehaene S. Levels of processing during non-conscious perception: a critical review of visual masking. Philos Trans R Soc Lond B Biol Sci. 2007; 362:857-875. [PubMed: 17403642]

Lamme VA. Why visual attention and awareness are different. Trends Cogn Sci. 2003; 7:12-18. [PubMed: 12517353]

Leber AB, Egeth HE. It's under control: top-down search strategies can override attentional capture. Psychon Bull Rev. 2006; 13(1):132-8. [PubMed: 16724780]

Lee YC, Lee JD, Boyle LN. The interaction of cognitive load and attention-directing cues in driving. Hum Factors. 2009; 51(3):271-80. [PubMed: 19750791]

Li Z. A saliency map in primary visual cortex. Trends in Cognitive Sciences. 2002; 6:9-16. [PubMed: 11849610]

Lin JY, Murray SO, Boynton GM. Capture of attention to threatening stimuli without perceptual awareness. Current Biology. 2009; 19:1118-1122. [PubMed: 19523828]

Mack, A.; Rock, I. Inattentional Blindness. Cambridge, MA: MIT Press; 1998.

McCormick PA. Orienting attention without awareness. J Exp Psychol Hum Percept Perform. 1997; 23(1):168-80. [PubMed: 9157183]

Merikle PM, Joordens S. Parallels between perception without attention and perception without awareness. Consciousness and Cognition. 1997; 6:219-236.

Moore CM, Egeth HE. Perception without attention: evidence of grouping under conditions of inattention. J Exp Psychol Hum Percept Perform. 1997; 23(2):339-352. [PubMed: 9103998]

Naccache L, et al. Unconscious masked priming depends on temporal attention. Psychol Sci. 2002; 13:416-424. [PubMed: 12219807]

Neisser, U. Cognitive Psychology. Appleton-Century-Crofts; New York: 1967.

O'Regan JK, Noe A. A sensorimotor account of vision and visual consciousness. Behav Brain Sci. 2001; 24:939-973. [PubMed: 12239892]

Santangelo V, Finoia P, Raffone A, Belardinelli MO, Spence C. Perceptual load affects exogenous spatial orienting while working memory load does not. Exp Brain Res. 2008; 184(3):371-82. [PubMed: 17763843]

Treisman AM. Preattentive processing in vision. Comput Vis Graph Image Proc. 1985; 31:156-177.

Tsuchiya N, Koch C. Continuous flash suppression reduces negative afterimages. Nat Neurosci. 2005; 8:1096-1101. [PubMed: 15995700]

Wyart V, Tallon-Baudry C. Neural dissociation between visual awareness and spatial attenton. J Neuroscience. 2008; 28:2667-2679.

Wolfe JM. Guided search 2.0: A revised model of visual search. Psychonomic Bulletin and Review. 1994; 1:202-238.

Zhaoping L. Attention capture by eye of origin singletons even without awareness - A hallmark of a bottom-up saliency map in the primary visual cortex. Journal of Vision. 2008; 8(5):1-18. 1. 

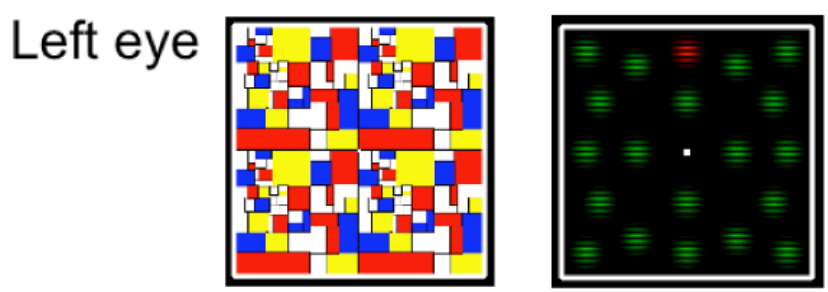

\section{Right eye \\ $2000 \mathrm{~ms}$}
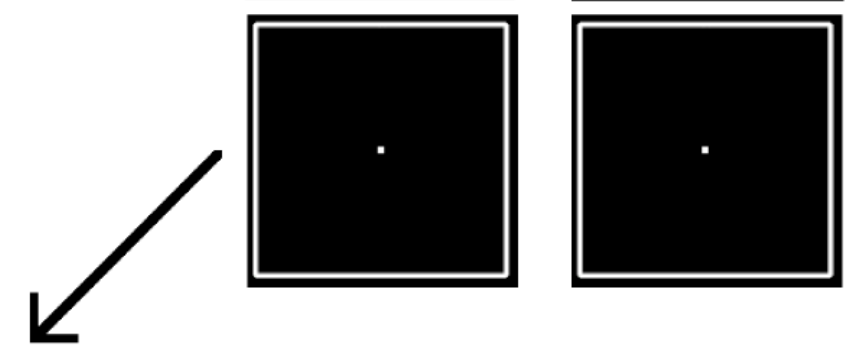

$250 \mathrm{~ms}$
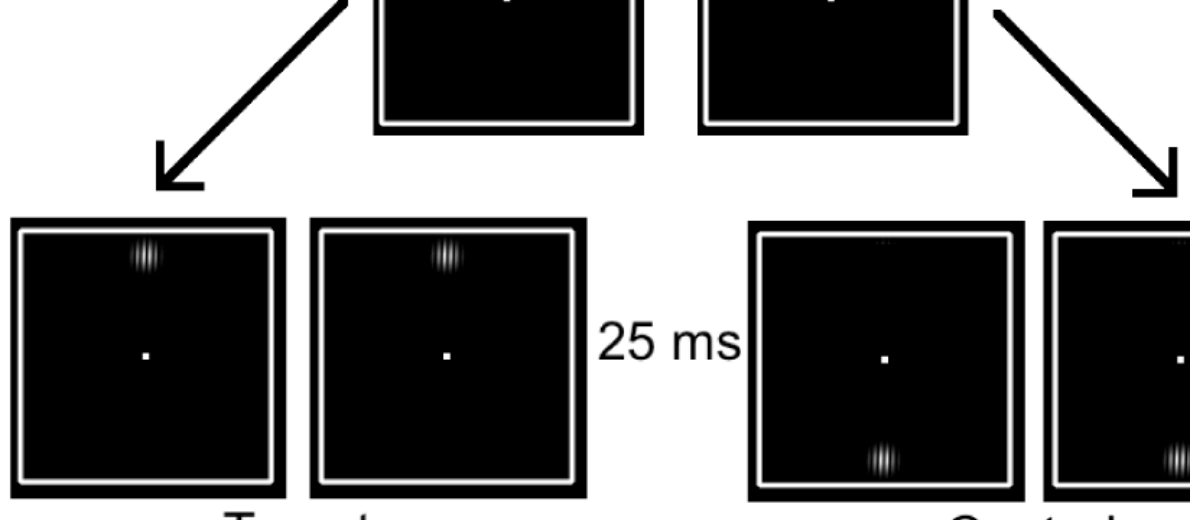

Target

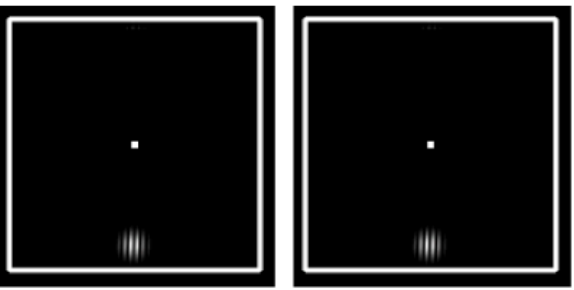

Control

Figure 1. Stimuli and procedures used in Experiment 1a

Participants viewed dichoptic images through a mirror stereoscope in a dark room. During the initial phase, a pop-out display of twenty horizontal Gabors appeared in one eye and gradually increased in contrast. Dynamic Mondrian patterns were presented in the other eye to suppress the pop-out display. After a blank inter-stimulus interval, a nearly vertical Gabor was presented at either the location of the unique red Gabor or the diametrically opposed control location. The test Gabor was rotated $1.5 \mathrm{deg}$ clockwise or counterclockwise, and the observer was required to press one of two buttons (2AFC) to indicate which orientation he or she perceived. At the end of every trial, subjects also reported whether or not the Mondrians effectively masked the colored Gabors. 


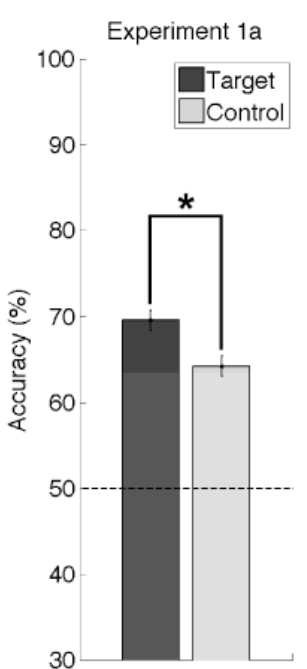

a.

Figure 2. Results of Experiments 1a and 1b

(a) Data averaged across seven subjects in Experiment 1a show that performance on the orientation discrimination task for the pop-out target location is significantly greater than that of the control location. (b) Data averaged across twelve subjects in Experiment $1 \mathrm{~b}$ indicate that performance on the orientation discrimination task for the pop-out target location is significantly greater than that of the control location. Error bars in all figures indicate standard errors across subjects. 

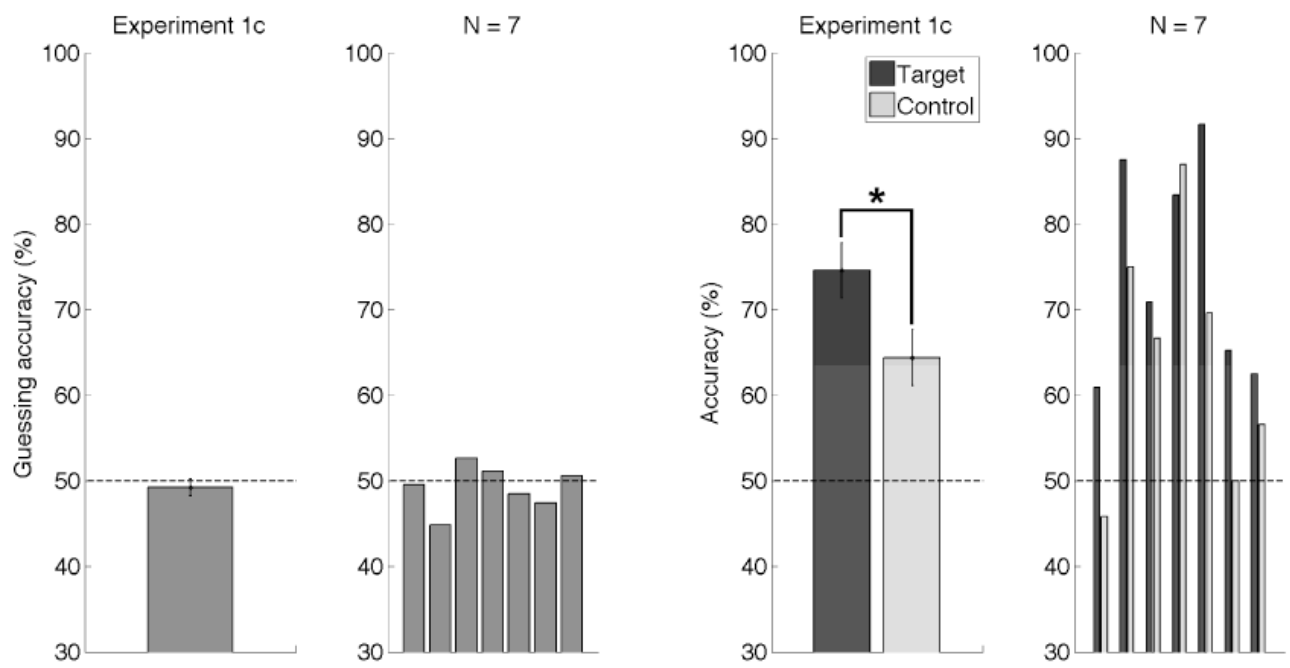

a.

b.

Figure 3. Results of Experiment 1c

(a) Data averaged across seven subjects show the percentage of trials in which subjects correctly guessed whether the pop-out display was present is not significantly different from chance. (b) Data averaged across seven subjects in Experiment 1c indicate that performance on the orientation discrimination task for the pop-out target location is significantly greater than that of the control location. 


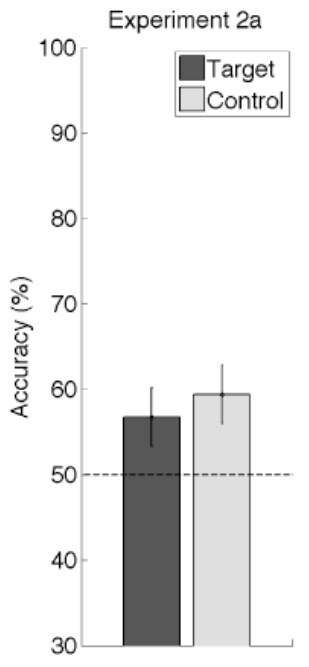

a.

Figure 4. Results of Experiment 2

(a) Data averaged across seven subjects in Experiment 2a indicate that performance on the orientation discrimination task for the pop-out target location is not significantly different from that of the control location. (b) Data averaged across five subjects in Experiment $2 b$ show that performance on the orientation discrimination task for the target (feature singleton) location is significantly different from that of the control location for the "attention available" (+Attention) blocks, but not for the "no attention" (-Attention) blocks. A significant interaction between block and location was found only at the $300 \mathrm{~ms}$ ISI. 